\title{
Análisis didáctico de una lección sobre proporcionalidad en un libro de texto de primaria con herramientas del enfoque ontosemiótico
}

\author{
Didactical analysis of a lesson on proportionality of a primary school \\ textbook using tools of the onto-semiotic approach
}

María Burgos*

ORCID iD 0000-0002-4598-7684

María José Castillo**

ORCID iD 0000-0002-8046-8927

Pablo Beltrán-Pellicer***

ORCID iD 0000-0002-1275-9976

Belén Giacomone ${ }^{* * * *}$

ORCID iD 0000-0001-6752-2362

Juan D. Godino ${ }^{* * * * *}$

ORCID iD 0000-0001-8409-0258

\begin{abstract}
Resumen
El libro de texto es un recurso ampliamente usado en la práctica de la enseñanza y, en consecuencia, es objeto de investigación educativa, aplicando diversas perspectivas teóricas. En este trabajo se ejemplifica el uso de las herramientas teóricas del Enfoque Ontosemiótico del conocimiento y la instrucción matemáticos para realizar el análisis didáctico de una lección sobre proporcionalidad de sexto curso de primaria (11-12 años de edad). Se considera que una lección refleja el proceso de instrucción planificado por el autor como medio para lograr el
\end{abstract}

\footnotetext{
* Doctora en Matemáticas por la Universidad de Almería (UAL). Profesora Contratada Doctora en el Departamento de Didáctica de la Matemática de la Universidad de Granada (UGR), España. Dirección postal: Facultad de Ciencias de la Educación. Campus Universitario de Cartuja, Granada, España, código postal 18071. E-mail: mariaburgos@ugr.es.

** Licenciada en Enseñanza de las Matemáticas (UCR). Profesora de la Universidad de Costa Rica (UCR) en la Facultad de Matemática, San Pedro, San José, Costa Rica. Dirección postal: Sede "Rodrigo Facio Brenes" Montes de Oca, San José Costa Rica, San José (Facultad de Matemáticas, código postal 11501-2060. E-mail: mariajosecastilloc.24@gmail.com.

*** Doctor por la Universidad Nacional de Educación a Distancia (UNED). Profesor asociado en la Universidad de Zaragoza, España. Dirección postal: Área de Didáctica de la Matemática, Facultad de Educación, c/ Pedro Cerbuna, 12, Zaragoza, España, código postal 50009. E-mail: pbeltran@unizar.es.

***** Doctora en Ciencias de la Educación por la Universidad de Granada (UGR). Profesora contratada en la Università degli Studi della Repubblica di San Marino (UNIRSM), Rep. San Marino. Dirección postal: Consiglio dei Sessanta, 99, Dogana, Rep. San Marino, código postal 47891. E-mail: belen.giacomone@gmail.com

***** Doctor por la Universidad de Granada (UGR). Catedrático (jubilado); Colaborador Extraordinario del Departamento de Didáctica de la Universidad de Granada, España. Dirección postal: Facultad de Educación, Granada, España, código postal 18071. E-mail: jgodino@ugr.es.
} 
aprendizaje del contenido por parte de los estudiantes potenciales del mismo. En consecuencia, se plantean las siguientes cuestiones que guían el análisis: 1) ¿Cuál es el grado de idoneidad didáctica del proceso de instrucción planificado? 2) ¿Cómo gestionar el uso del texto para incrementar la idoneidad del proceso? El método de análisis implementado permite obtener conocimientos didáctico-matemáticos que pueden ayudar al profesor a tomar decisiones sobre las posibilidades y limitaciones de los libros de texto cuando se asumen los postulados socioculturales sobre el aprendizaje matemático.

Palabras clave: Análisis didáctico. Libro de texto. Enfoque ontosemiótico. Idoneidad didáctica.

\begin{abstract}
The textbook is a widely used resource in the practice of teaching and, consequently, is a subject of educational research considered from various theoretical perspectives. In this paper, we apply the theoretical tools from the onto-semiotic approach to mathematical knowledge and instruction to perform the didactical analysis of a lesson on proportionality for elementary school (sixth grade; 11-12 years old). The selected lesson allows us to reflect on the instructional process planned by the textbook authors as a means to achieve the students' learning. The following questions guide the textbook analysis: 1) What is the degree of didactical suitability of the planned instructional process? 2) How should we manage the use of the textbook to increase the process suitability? The method of analysis implemented allows us to obtain the didactic-mathematical knowledge that may help the teacher in making decisions about the textbook possibilities and limitations, when assuming the sociocultural postulates for mathematical learning.
\end{abstract}

Keywords: Didactical analysis. Textbook. Onto-semiotic approach. Didactical suitability.

\title{
1 Introducción
}

El análisis de libros de texto es un tema relevante tanto para la práctica de la enseñanza como para la investigación en educación matemática. La revisión realizada por Fan, Zhu y Miao (2013) revela que se han hecho progresos importantes, en las últimas décadas, en la investigación sobre libros de textos de matemáticas, abarcando temáticas tales como: papel de los libros en la enseñanza y el aprendizaje; análisis de las características de los libros y comparación entre dos o más series de libros; uso de los libros por parte de los profesores y los estudiantes; otros temas, como las relaciones entre los libros y el rendimientos de los estudiantes, libros electrónicos etc.

Según American Association for the Advancement of Science (2000) el libro de texto es considerado, por muchos profesores, como su principal guía en la implementación del currículo. El Proyecto 2061 de esta asociación comenzó la evaluación de libros de texto basándose en tres proposiciones: los libros pueden ser claves para mejorar la educación matemática, la calidad de los libros debe juzgarse en base a su efectividad para ayudar a los estudiantes a lograr objetivos de aprendizaje de matemáticas establecidos en los programas de estudio, y tercero, es necesario un análisis profundo que contemple no solo el contenido abordado en un libro con el fin de evaluar el potencial del libro para que los estudiantes lo aprendan. 
En este trabajo desarrollamos un método para realizar el análisis en profundidad y amplitud de las características de una lección de un libro de texto, que aborda el estudio de la proporcionalidad en el último curso de educación primaria, aplicando herramientas del Enfoque Ontosemiótico (EOS) (GODINO, 2017; GODINO; BATANERO; FONT, 2007). El método descrito complementa y amplia los aportes presentados en Godino, Font y Wilhelmi (2006), Font y Godino (2006) y otros trabajos realizados en el marco del EOS (GEA, 2014; ORTIZ, 1999). Una lección de un libro de texto, la vamos a considerar como un proceso de instrucción (potencial o planificado) que se compone de la secuencia de prácticas matemáticas y didácticas que propone el autor para el estudio del tema en cuestión, en nuestro caso la proporcionalidad en sexto curso de primaria.

La secuencia de actividades que se proponen en un libro, bien como ejemplos introductorios de conceptos o procedimientos, o como problemas y ejercicios a realizar por los estudiantes, no solo incluyen el desarrollo del contenido matemático (faceta epistémica, esto es, conocimientos institucionales planificados), sino que, también, ponen en juego conocimientos previos que los estudiantes deben tener para que puedan involucrarse y comprender el desarrollo de la lección. Esto quiere decir que en el análisis de una lección también se debe tener en cuenta cómo se gestionan los conocimientos previos, lo cual involucra un aspecto de las facetas cognitiva (significados personales).

En el libro de texto se describen los problemas matemáticos que se deben resolver aplicando el modelo matemático correspondiente, los ejemplos introductorios, definiciones, explicaciones, justificaciones; también se indica lo que deben hacer los alumnos, y en algunos casos cómo hacerlo (trabajando en equipo o individualmente). Con frecuencia se incluyen, también, elementos motivadores (ilustraciones, contextos de aplicación) y referencia a recursos didácticos complementarios (manipulativos, applets etc.).

En nuestro caso, el análisis didáctico se orienta a identificar elementos que pueden ser potencialmente conflictivos, desde el punto de vista de los conocimientos matemáticos pretendidos, y del proceso instruccional que se propone. Esta información puede ser útil para el profesor que use el libro como recurso, a fin de tener en cuenta tales conflictos y prever posibles soluciones.

Este trabajo se organiza en las siguientes secciones: (a) Marco teórico, problema y método; (b) Conocimientos didáctico-matemáticos sobre proporcionalidad; criterios de idoneidad; (c) Descripción general de la lección sobre proporcionalidad; (d) Análisis de las facetas epistémica y cognitiva; (e) Faceta instruccional; (f) Síntesis de hechos didácticos significativos; (g) Comentarios finales e implicaciones. 


\section{Marco teórico, problema y método}

Considerando la lección de un libro de texto como un proceso instruccional (previsto o planificado), las distintas herramientas teóricas elaborados por el Enfoque Ontosemiótico (EOS) del conocimiento y la instrucción matemáticos (GODINO, 2017; GODINO; BATANERO; FONT, 2007; GODINO; BATANERO; FONT, 2019a) se pueden aplicar para realizar un análisis sistemático de lecciones de los libros de texto. La Teoría de la Idoneidad Didáctica (TID) (BREDA; FONT; PINO-FAN, 2018; BREDA; PINO-FAN; FONT, 2017; GODINO, 2013) ayuda a formular el problema del análisis didáctico de los libros de texto en términos de caracterizar la idoneidad de las trayectorias didácticas propuestas en los mismos e identificar posibles cambios para mejorar los aprendizajes pretendidos. Las diferentes facetas, componentes e indicadores de idoneidad pueden servir de base para la elaboración de una Guía de análisis de libros de textos en la cual se reflejen las diversas variables a tener en cuenta.

La emisión de juicios razonados sobre la idoneidad de un proceso instruccional requiere tener en cuenta los diversos significados de los objetos matemáticos de los contenidos implicados y la identificación de la trama de objetos y procesos que se ponen en juego en las prácticas matemáticas que se realizan. Estos análisis se apoyan en las nociones de significado pragmático y configuración ontosemiótica (GODINO et al., 2017) las cuales ayudan a identificar conflictos epistémicos y cognitivos potenciales en el desarrollo de la lección.

La noción de configuración didáctica proporciona criterios para descomponer el proceso de instrucción en unidades de análisis, en las cuales se identifican hechos didácticos significativos (GODINO et al., 2014b) relativos a las diferentes facetas, componentes e indicadores de idoneidad. En Godino, Contreras y Font (2006) la noción de configuración didáctica (CD) se asocia a una situación problema y tiene en cuenta los conocimientos implicados en la solución, los recursos usados y las acciones docentes y discentes que se realizan. En este trabajo consideramos que el foco de una CD puede ser otro tipo de objetos primarios, por ejemplo, la introducción de un concepto o un procedimiento.

La herramienta dimensión normativa (GODINO et al., 2009) nos lleva a pensar en el tipo de contrato didáctico que, de manera más o menos explícita, induce el autor del texto, los condicionamientos curriculares bajo los cuales se desarrolla el proceso instruccional, y cualquier otra restricción, hábito o comportamiento que pueda influir en los aprendizajes alcanzables. 
Suponiendo que un profesor ha tomado la decisión de usar el libro de texto como recurso para apoyar su enseñanza y el aprendizaje de los estudiantes, y teniendo en cuenta el estado de los conocimientos didáctico-matemáticos sobre el tema, las cuestiones que nos planteamos son:

\section{1) ¿Cuál es el grado de idoneidad didáctica del proceso de instrucción planificado?}

Esto implica analizar los significados del contenido que se incluyen, los tipos de situaciones-problemas, las representaciones (sus conversiones y tratamientos), los conceptos, procedimientos, proposiciones y argumentos que se aportan y los que no.

También, implica identificar los conocimientos previos que se van requiriendo a lo largo del proceso, los recursos didácticos que se proponen y los modos de interacción.

Estos análisis deben permitir identificar conflictos epistémicos (relativos a los significados y objetos institucionales puesto en juego en la lección), conflictos cognitivos potenciales (relacionados con los conocimientos previos requeridos) y conflictos instruccionales (modos de interacción y uso de recursos). Las variables a tener en cuenta en el análisis de un texto vienen, por tanto, determinadas por las facetas, componentes y criterios de idoneidad que propone la Teoría de la Idoneidad Didáctica.

2) ¿Cómo gestionar el uso del texto para incrementar la idoneidad del proceso de estudio?

La consideración del texto como un proceso de instrucción potencial o planificado por el autor del libro, que puede servir de apoyo al profesor para diseñar e implementar un proceso de instrucción efectivo, permite aplicar las herramientas de análisis didáctico del EOS para valorar la idoneidad didáctica de dicho proceso, identificar posibles conflictos de significado y potenciales mejoras. El profesor que use el texto como recurso podría tener en cuenta los resultados de nuestro análisis para optimizar el aprendizaje de sus alumnos. El análisis descriptivo de cómo usan el libro de texto los profesores y los estudiantes en las clases reales se puede apoyar en las variables ligadas a las facetas y componentes de la TID, mientras que las variables derivadas de los indicadores de idoneidad puede orientar la emisión de heurísticas de cómo se deberían usar los libros de texto.

\section{Conocimientos didáctico-matemáticos sobre proporcionalidad. Criterios de idoneidad}

De acuerdo con el marco teórico del EOS, la reconstrucción del significado de referencia global de un objeto matemático, en nuestro caso la proporcionalidad, debe tener en cuenta los conocimientos sobre las diferentes dimensiones implicadas en los procesos de 
enseñanza y aprendizaje. En esta sección, incluimos una síntesis de conocimientos didácticos que servirán de referencia para valorar la idoneidad didáctica del libro de texto.

\subsection{Significados de la proporcionalidad}

Desde la perspectiva epistémica, la proporcionalidad puede ser considerada según cuatro enfoques:

Informal-cualitativo, centrado en la comparación multiplicativa de las cantidades que intervienen en los problemas y en la comparación perceptiva, por ejemplo, de la semejanza de formas geométricas.

Geométrico, centrado en las razones y proporciones entre segmentos, escalas y semejanza de figuras. Las tareas relacionadas con escalas, ampliaciones y reducciones de figuras preservando la forma, permiten avanzar desde la aproximación intuitiva y cualitativa (pre-proporcional) a un significado cuantitativo esencialmente multiplicativo (proporcional).

Aritmético, centrado en la noción de razón y proporción. Una razón establece una comparación multiplicativa entre un par ordenado de cantidades de magnitudes (homogéneas o heterogéneas) cada una de las cuales viene expresada mediante un número real y una unidad de medida. Una proporción es la igualdad de dos razones. El enfoque aritmético del estudio de la proporcionalidad predomina en la mayoría de las propuestas curriculares e investigaciones, distinguiéndose, esencialmente, dos categorías de problemas:

- Problemas de comparación. En un problema de este tipo, se dan cuatro valores, relacionados de manera multiplicativa dos a dos, formando dos razones. El procedimiento de resolución es totalmente aritmético: la proporción es una relación de igualdad entre dos razones, $\frac{a}{b}=\frac{c}{d}$, donde a, b, c y d son números enteros cualesquiera, y las razones a/b y c/d son relaciones multiplicativas entre los números $\mathrm{a}, \mathrm{b}$ y c, $\mathrm{d}$, respectivamente.

- Problemas de valor faltante. En un problema de valor faltante, la proporción es una relación de igualdad entre dos razones, en la que uno de los términos es un valor desconocido (valor faltante). Por ejemplo, $\frac{a}{b}=\frac{c}{x}$, donde $\mathrm{a}, \mathrm{b}$ y c son números enteros conocidos y $x$ es el valor que se pretende determinar.

Algebraico, centrado en la noción de función lineal. Desde el enfoque funcional, se asume que "el razonamiento proporcional supone un tipo de razonamiento en un sistema de dos variables entre las que existe una relación funcional lineal que permite obtener 
conclusiones sobre una situación o fenómeno que puede ser caracterizado por una razón constante" (KARPLUS; PULOS; STAGE, 1983, p. 192). El modelo matemático que responde a esta situación es una función $y=k x$, donde $k$ es la razón constante unitaria o constante de proporcionalidad.

Para Lamon (2007) el razonamiento proporcional reside en el reconocimiento de la razón constante entre elementos del mismo espacio de medida y el reconocimiento de la relación escalar entre diferentes espacios de medida. Supone la habilidad de reconocer una relación multiplicativa entre dos cantidades, así como la capacidad de extender la misma relación a otros pares de cantidades.

Recientemente, Godino et al. (2017) distinguen tres tipos de significados del objeto proporcionalidad: aritmético, proto-algebraico y algebraico-funcional, basándose en la aplicación de los niveles de algebrización (GODINO et al., 2014a) a los sistemas de prácticas ligados a las tareas de proporcionalidad.

El significado aritmético (nivel 0 de algebrización) se caracteriza por la aplicación de procedimientos de cálculo aritméticos (multiplicación, división). En la práctica intervienen valores numéricos particulares y se aplican operaciones aritméticas sobre dichos valores; no intervienen objetos y procesos algebraicos. El significado proto-algebraico está centrado en la noción de proporción, de manera que el reconocimiento del valor unitario en un procedimiento de reducción a la unidad, y el uso de representaciones diagramáticas de soluciones se pueden calificar de proto-algebraicas de nivel 1. Por otro lado, la solución de un problema de valor perdido basada en el uso de las razones y proporciones, involucra una incógnita y el planteamiento de una ecuación; la actividad de algebrización que se realiza en este caso es proto-algebraica de nivel 2, según el modelo de Godino et al. (2014a), ya que la incógnita aparece despejada en un miembro de la ecuación que se establece $(A x=B)$.

Como señala Wilhelmi (2017a, p. 5), en Educación Primaria, para organizar situaciones cotidianas que involucran la proporcionalidad se recurre, en muchas ocasiones, a la construcción de tablas que permiten la organización eficaz de la información y facilitan la obtención del valor que corresponde a la unidad (nivel 1 de algebrización). Esta introducción suele ser obviada en el desarrollo de la proporcionalidad en Educación Secundaria que contempla, casi exclusivamente, un uso técnico de la regla de tres (nivel 2 de algebrización) o una interpretación a partir de la fórmula de función lineal (nivel 3 de algebrización). 


\subsection{Orientaciones curriculares}

La proporcionalidad y porcentajes se contemplan en diferentes bloques temáticos dentro del currículo español (MECD, 2014) para la educación primaria (Cuadro 1).

\begin{tabular}{|c|c|c|}
\hline Contenidos & $\begin{array}{l}\text { Criterios de } \\
\text { Evaluación }\end{array}$ & Estándares de aprendizaje evaluables \\
\hline \multicolumn{3}{|c|}{ Bloque 2. Números } \\
\hline $\begin{array}{l}\text { Porcentajes y } \\
\text { proporcionalidad. Porcentajes: } \\
\text { Expresión de partes utilizando } \\
\text { porcentajes. Correspondencia } \\
\text { entre fracciones sencillas, } \\
\text { decimales y porcentajes. } \\
\text { Aumentos y disminuciones } \\
\text { porcentuales. } \\
\text { Proporcionalidad directa. La } \\
\text { Regla de tres en situaciones de } \\
\text { proporcionalidad directa: ley } \\
\text { del doble, triple, mitad. }\end{array}$ & $\begin{array}{l}\text { 7. Iniciarse en el uso } \\
\text { de los de porcentajes } \\
\text { y la proporcionalidad } \\
\text { directa para } \\
\text { interpretar e } \\
\text { intercambiar } \\
\text { información y } \\
\text { resolver problemas en } \\
\text { contextos de la vida } \\
\text { cotidiana. }\end{array}$ & $\begin{array}{l}\text { 7.4. Usa la regla de tres en situaciones de } \\
\text { proporcionalidad directa: ley del doble, } \\
\text { triple, mitad, para resolver problemas de la } \\
\text { vida diaria. } \\
\text { 7.5. Resuelve problemas de la vida cotidiana } \\
\text { utilizando porcentajes y regla de tres en } \\
\text { situaciones de proporcionalidad directa, } \\
\text { explicando oralmente y por escrito el } \\
\text { significado de los datos, la situación } \\
\text { planteada, el proceso seguido y las } \\
\text { soluciones obtenidas. }\end{array}$ \\
\hline \multicolumn{3}{|c|}{ Bloque 4. Geometría } \\
\hline $\begin{array}{l}\text { La representación elemental } \\
\text { del espacio, escalas y gráficas } \\
\text { sencillas. }\end{array}$ & & $\begin{array}{l}\text { 1.4. Realiza escalas y gráficas sencillas, para } \\
\text { hacer representaciones elementales en el } \\
\text { espacio. } \\
\text { 1.7 Realiza ampliaciones y reducciones } \\
\text { 6.1 Comprende y describe situaciones de la } \\
\text { vida cotidiana, e interpreta y elabora } \\
\text { representaciones espaciales (planos, croquis } \\
\text { de itinerarios, maquetas...), utilizando las } \\
\text { nociones geométricas. }\end{array}$ \\
\hline
\end{tabular}

Cuadro 1 - Contenidos, criterios de evaluación y estándares de aprendizaje evaluables Fuente: elaborado por el autor

En el Cuadro 1 se recogen las referencias a proporcionalidad aritmética y geométrica (escalas) en dicho currículo. Por otro lado, de acuerdo con los Principios y Estándares del NCTM (2000) la proporcionalidad es un elemento integrador importante que conecta muchos temas matemáticos estudiados en los grados 6-8. Involucra "reconocer cantidades que están relacionadas proporcionalmente y usar números, tablas, gráficos y ecuaciones para pensar sobre las cantidades y sus relaciones" (NCTM, 2000, p. 217).

\subsection{Faceta cognitiva}

Desde una perspectiva psicológica-cognitiva, según Inheler y Piaget (1958), el razonamiento proporcional es una relación de segundo orden que implica una relación de equivalencia entre dos razones. Requiere del uso de un razonamiento hipotético deductivo que permite al sujeto utilizar una relación matemática (razón) y a partir de ésta deducir una segunda relación también matemática (proporción). 
Según muestran diversas investigaciones, los problemas que involucran números naturales pequeños, aquellos en los que aparecen relacionados los primeros o segundos términos de una razón y en los que existe una relación de divisibilidad entre sus términos, resultan más fáciles para los alumnos (KARPLUS; PULOS; STAGE, 1983; TURNAIRE; PULOS, 1985).

El carácter tanto longitudinal como tras versal de la proporcionalidad en el currículo está justificado por la diversidad de contextos en los que tiene aplicación (WILHELMI, 2017a). El razonamiento proporcional es esencial para que el individuo pueda desenvolverse con fluidez en su día a día, y es el fundamento de diversos contenidos científico-artísticos del currículo escolar. Es importante que se dote de sentido al contenido matemático de la proporcionalidad mostrando la utilidad de su aprendizaje a través de situaciones próximas al contexto sociocultural del alumnado.

Por otro lado, la importancia de la proporcionalidad en el campo del early algebra (WILHELMI, 2017b) y el desarrollo de la "algebrización de la proporcionalidad" (GASCÓN; BOSCH; RUIZ-MUNZÓN, 2017) aparece como problema crucial en la naturaleza del álgebra escolar (GODINO; BURGOS, 2017).

\subsection{Faceta instruccional}

Desde una perspectiva instruccional, diversos autores proponen anticipar una aproximación informal, intuitiva y cualitativa al concepto de razón y proporción, previa a su formalización y algoritmización; promover estrategias de construcción progresiva del razonamiento proporcional que permita al alumno avanzar desde un conocimiento de estructura aditiva, hacia un conocimiento cuantitativo de estructura multiplicativa (FERNÁNDEZ, 2001; FIOL; FORTUNY, 1990; STREEFLAND, 1985).

Un uso indiscriminado de la regla de tres puede llevar a no desarrollar un razonamiento proporcional adecuado, $\mathrm{y}$, en particular, a no distinguir situaciones de proporcionalidad de aquellas que no lo son (FERNÁNDEZ; LLINARES, 2012). Por esto, antes de presentar este algoritmo a los estudiantes, se les debería ofrecer la oportunidad de desarrollar y argumentar sus propias estrategias.

En Aroza, Godino y Beltrán-Pellicer (2016) se incluye un sistema de indicadores de idoneidad didáctica específicos para los procesos de instrucción sobre el tema de la proporcionalidad, inferidos a partir de una revisión de las investigaciones sobre el tema. 


\section{Descripción general de la lección sobre proporcionalidad}

La lección que se analiza es la unidad 8 (Porcentajes y proporcionalidad) del libro de Ferrero et al. (2015) ${ }^{1}$ de sexto curso de primaria. La lección comienza con una sección en la que se describe una situación de uso del doble, triple y mitad de cantidades de peso y valor, en un contexto de preparación de una receta de cocina. Puede ser un contexto que motiva el estudio de las relaciones de proporcionalidad entre magnitudes, pero no se usa aquí con esa finalidad. Seguidamente se recuerda el concepto de porcentaje, como una forma de escritura de los números racionales y se aplica para hallar el porcentaje de una cantidad. El concepto de porcentaje y su aplicación se vuelve a considerar al final de la lección.

En las dos primeras páginas de la unidad se introducen las magnitudes directamente proporcionales; de la 114 a 115 , la regla de tres; de la 116 a 117, el tanto por ciento o porcentajes; en las 118 y 119, cálculo del tanto por ciento de una cantidad; 120 y 121, repaso de la unidad, en la que se incluye una sección de recuerdo (definiciones de magnitudes directamente proporcionales, reducción a la unidad, regla de tres y cálculo de porcentajes).

En la página 122 se incluye una sección titulada Aprendo a resolver problemas, donde se da una pauta a seguir para resolver un problema de proporcionalidad simple directa, aplicando el método de la regla de tres; se incluyen, también, dos nuevos problemas para resolver. La lección termina con una nueva tarea titulada El desafío. Tarea competencial, en la que se plantea una situación de preparación de limonadas y bizcochos, similares a las tratadas previamente. En esta misma sección se incluye una tarea abierta en la que, siguiendo criterios de proporcionalidad, se debe preparar una receta para nueve personas con ingredientes que se deben elegir libremente.

En este artículo centramos el análisis en la parte de proporcionalidad, sin considerar la sección de porcentajes. En el siguiente apartado definimos las unidades de análisis (configuraciones didácticas) en que dividimos las secciones del texto que analizamos.

\footnotetext{
${ }^{1}$ El análisis didáctico que se realiza del libro es parcial, ya que solo se aplica a partes selectivas de una sola lección; no se analizan, con detalle, algunas secciones de la lección, ni tampoco las orientaciones metodológicas que los autores incluyen en el libro del profesor. Los juicios valorativos que se emiten, teniendo en cuenta los criterios de idoneidad didáctica específicos sobre la enseñanza de la proporcionalidad, se refieren a un proceso de instrucción que un docente hipotético pudiera implementar haciendo un uso plausible del libro, el cual puede diferir de las intenciones de los autores.
} 


\section{Análisis de las facetas epistémica y cognitiva}

En esta sección centramos la atención en ocho unidades de análisis o configuraciones didácticas. En cada una de ellas describimos, en primer lugar, las prácticas matemáticas operativas y discursivas que se proponen e identificamos los objetos matemáticos que intervienen en las mismas. Seguidamente, describimos los principales procesos matemáticos, y los conflictos epistémicos y cognitivos que se manifiestan, teniendo en cuenta el marco de referencia y en particular el modelo epistémico y didáctico de estudio de la proporcionalidad que previamente hemos elaborado.

\subsection{Configuración 1 (Conceptualización): Definición de magnitudes directamente proporcionales}

\section{Prácticas y objetos intervinientes}

Se indica la relación entre distintas cantidades de compra de kilogramos de café y el precio que se debe pagar. Se describe la práctica social de que al comprar el doble, triple, cuádruple etc., (respectivamente, la mitad, tercio etc.) el precio que se debe pagar es el doble, triple, etc. La situación se ilustra con un ejemplo (Figura 1) en el que se supone que el precio de un $\mathrm{kg}$ de café es $12 €$, usando un registro tabular para indicar la serie de números directamente proporcionales: valores numéricos de cantidades de café y sus correspondientes valores numéricos del precio que se paga. De manera diagramática se indica que, si la cantidad de café se multiplica por 2, 3 o 4, el precio del café se multiplica también por 2, 3 o 4.

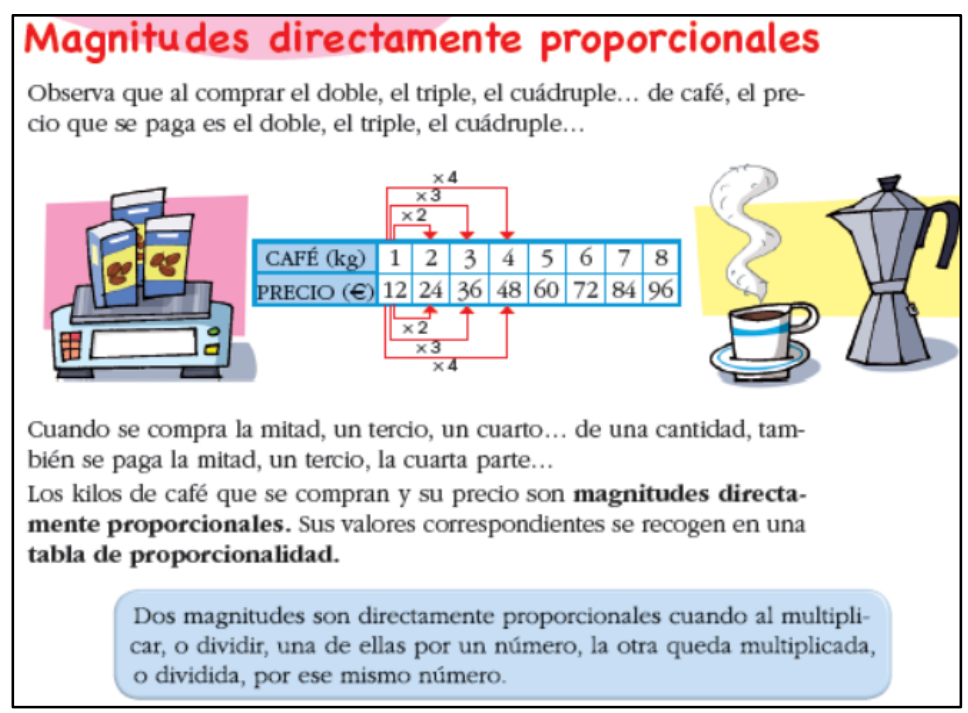

Figura 1 - Magnitudes directamente proporcionales Fuente: FERRERO et al. (2015, p. 112) 
La relación funcional lineal que se establece en esta situación se presenta como un ejemplar prototípico de un tipo de relación que se nombra como magnitudes directamente proporcionales. Se introduce la expresión tabla de proporcionalidad para referirse a la representación tabular de la relación de proporcionalidad directa entre magnitudes.

Intervienen los conceptos de magnitud, múltiplo (doble, triple, cuádruple etc.) o divisor (mitad, tercio, cuarta parte etc.), que se suponen conocidos (conceptos previos).

Procesos

- Definición/conceptualización:

- La configuración tiene por objetivo presentar una definición de magnitudes directamente proporcionales (MDP), generalizando el criterio usado en el ejemplo (Figura 2) para cualquier tipo de magnitudes:

Dos magnitudes son directamente proporcionales cuando al multipli-
car, o dividir, una de ellas por un número, la otra queda multiplicada,
o dividida, por ese mismo número.

Figura 2 - Definición de magnitudes directamente proporcionales Fuente: FERRERO et al. (2015, p. 112)

- El objeto tabla de proporcionalidad se introduce haciendo referencia al ejemplo de tabla de dos filas con los valores numéricos de las medidas de las cantidades de las dos magnitudes.

- Generalización:

- El criterio general de linealidad de la relación funcional, $y=\mathrm{k} x$, se evoca mediante puntos suspensivos.

- La definición de MDP es general para cualquier par de magnitudes, no solo para el caso de la compra de café.

- La interpretación de la tabla de proporcionalidad conlleva un proceso de generalización de la relación de proporcionalidad. Es decir, un aumento multiplicativo en una de las filas se corresponde con el mismo aumento multiplicativo en la otra.

- Representación/interpretación:

- Además del lenguaje natural se usan las representaciones, tabular y diagramática.

- En el diagrama, el doble, triple, cuádruple son representados por los símbolos $\times 2, \times 3, \times 4$. Se supone que los números situados en la primera fila, en cada columna, se corresponden con los números situados en la segunda fila. 


\section{Conflictos epistémicos}

No se razona que la situación de compra-venta es una práctica social sujeta a convenios y reglas, obviando posibles situaciones de variación del precio unitario cuando las cantidades son relativamente importantes. La relación funcional lineal entre las magnitudes referidas, en principio, se establece entre cualquier par de cantidades, no solo en los ocho casos representados.

Igual ocurre cuando las cantidades no son múltiplos sino divisores, mitad, tercio, cuarto. El carácter general de la secuencia de valores se indica meramente con puntos suspensivos.

\section{Conflictos cognitivos potenciales}

Se suponen conocidos los conceptos de magnitud, unidad de medida, cantidad y valor numérico de las medidas, lo cual puede ser una fuente de conflictos si el lector no está familiarizado con ellos.

También, pueden requerir explicación los convenios de representación de la función mediante la tabla bidimensional y los diagramas indicativos del incremento de las variables. Una mirada superficial a los diagramas puede llevar a pensar que la presencia de una tabla bidimensional supone la existencia de una relación de proporcionalidad.

\subsection{Configuración 2 (ejercitación)}

\section{Prácticas y objetos}

La primera situación-problema plantea dos enunciados de reconocimiento de magnitudes directamente proporcionales, ilustradas mediante diagramas de correspondencia de tres pares de cantidades (Figura 3, parte 1). Una relación, sí es de proporcionalidad, la otra no.

La segunda situación-problema mostrada en la misma Figura 3 se enuncia a partir de los datos en una tabla definida en un contexto del mundo real.

Por último, la tercera situación-problema (Figura 3) pretende el reconocimiento de tablas de proporcionalidad con datos no contextualizados. 


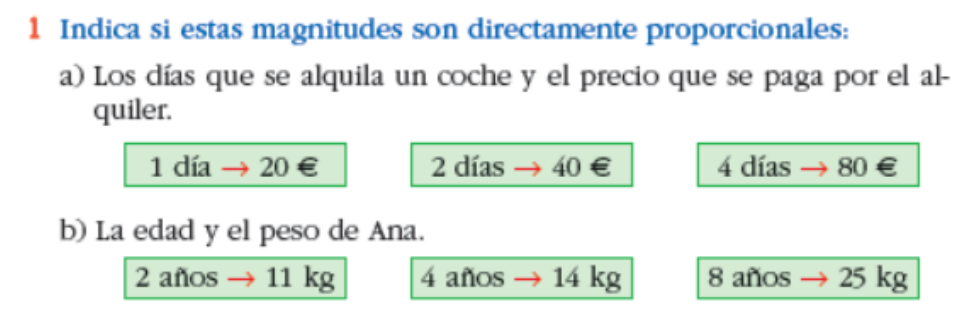

2 Estas son las tarifas de un vivero:

\begin{tabular}{|c|c|c|c|}
\hline ROSALES & 3 & 6 & 12 \\
\hline PRECIO $(\Theta)$ & 24 & 40 & 75 \\
\hline
\end{tabular}

- ¿Es una tabla de proporcionalidad? ¿Por qué?

3 ¿Son de proporcionalidad estas tablas?:

a) \begin{tabular}{|c|c|c|c|}
\hline 3 & 6 & 9 & 12 \\
\hline 9 & 15 & 21 & 36 \\
\hline
\end{tabular}$\quad$ b) \begin{tabular}{|c|c|c|c|}
\hline 2 & 4 & 8 & 20 \\
\hline 10 & 20 & 40 & 100 \\
\hline
\end{tabular}

Figura 3 - Ejercitación. Tablas de proporcionalidad Fuente: FERRERO et al. (2015, p. 112)

\section{Procesos}

- Ejercitación: reconocimiento de situaciones de aplicación del criterio de definición de la función de proporcionalidad directa entre magnitudes y del concepto de tabla de proporcionalidad.

- Representación/ interpretación: los diagramas de correspondencia y tabular como medios de expresión de la función lineal.

\section{Conflictos epistémicos}

- Se usa la expresión tabla de proporcionalidad como sinónimo de serie de números proporcionales; se presentan como tales, tablas con 3 o 4 columnas, sin indicar que la serie es ilimitada.

- No se hace explícito que la razón entre las cantidades que se corresponden debe ser siempre la misma: constante de proporcionalidad. Al no especificar esta condición de regularidad se puede estar generando un obstáculo didáctico.

\subsection{Configuración 3 (procedimental). Reducción a la unidad}

\section{Prácticas y objetos}

Se comienza describiendo, de manera general, el procedimiento de reducción a la unidad para resolver problemas de valor perdido en situaciones de proporcionalidad directa (Figura 4); sin embargo, no se presentan previamente los problemas que se quieren resolver. Seguidamente, se muestra la aplicación del procedimiento a partir de un ejemplo concreto. 
Si doce botellas contienen dieciocho litros de agua, ¿cuál es la capacidad de siete botellas?

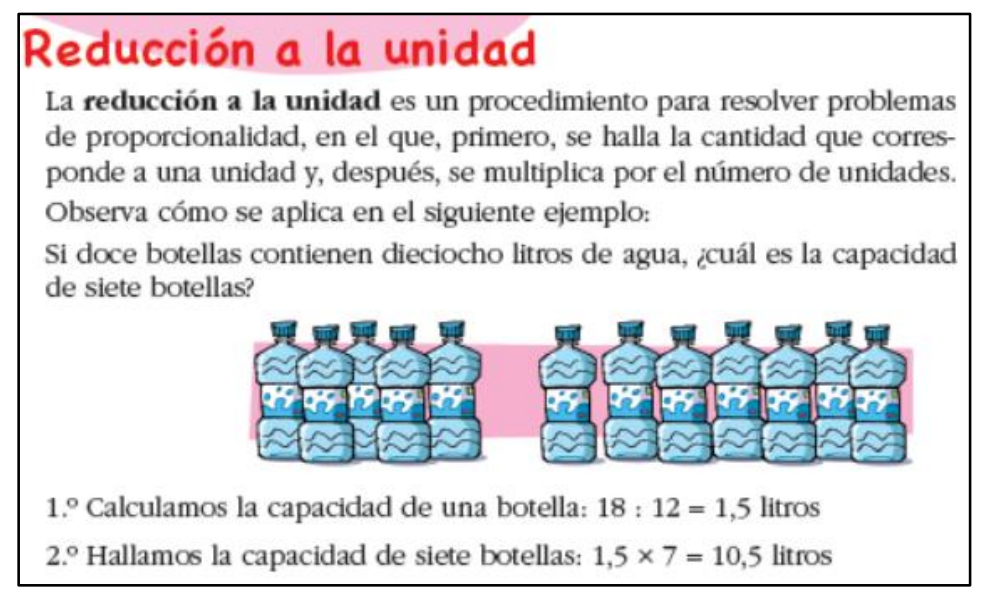

Figura 4 - Reducción a la unidad Fuente: FERRERO et al. (2015, p. 113)

\section{Procesos}

- Algoritmización: fijación de una regla para resolver problemas de proporcionalidad: primero se halla el valor que corresponde a la unidad, después se multiplica por el número de unidades.

- Representación/interpretación: se usa el lenguaje natural para describir el algoritmo; la igualdad de las capacidades de las botellas se asume visualmente con la interpretación del dibujo.

- Particularización: la regla general que describe el procedimiento inicialmente se ejemplifica con el caso particular de las capacidades de las botellas de agua.

\section{Conflictos epistémicos}

- Ausencia de argumentación/ justificación del procedimiento. No se indica que todas las botellas son iguales (condición de regularidad), razón por la cual para calcular la capacidad de una botella hay que dividir por 12 (concepto de división), y para calcular la capacidad de 7 hay que multiplicar por 7 .

- Se hace un uso incorrecto del signo igual, como resultado de una operación aritmética $(18: 12=1,5)$ y como cantidad de magnitud (1,5 litros). 


\subsection{Configuración 4 (ejercitación)}

\section{Prácticas y objetos}

Se proponen dos problemas de valores perdidos formulados de manera tabular con contexto (Figura 5). Al decir que se trata de tablas de proporcionalidad se está expresando que las relaciones entre las dos magnitudes indicadas son de proporcionalidad directa.

1 Copia y completa en tu cuaderno estas tablas de proporcionalidad:
\begin{tabular}{|c|c|c|c|c|c|c|c|}
\hline N. ${ }^{\circ}$ DE CAJAS & 5 & 1 & 2 \\
\hline N. ${ }^{\circ}$ DE NARANJAS & 10 & & \\
\hline
\end{tabular}

Figura 5 - Tablas de proporcionalidad incompletas Fuente: FERRERO et al. (2015, p. 113)

Mediante las tareas previas se supone que el estudiante no tendrá dificultades para interpretar el enunciado, en el sentido de que se requiere hallar el número de naranjas que caben en, primero en una caja (la quinta parte, es decir 2 naranjas), y después el doble de la quinta parte $(2 \times 2=4$, es decir 4 naranjas $)$.

\section{Procesos}

Tiene una orientación algorítmica, procedimental. Se trata de adquirir destreza en la aplicación de la rutina del método de reducción a la unidad, así como de la interpretación de la representación esquemática-tabular de los problemas de valor perdido en las relaciones de proporcionalidad.

\section{Conflictos potenciales}

Habría que enfatizar la presencia de una relación funcional, no reducida a dos o tres casos. Además, sería necesario discutir la condición de regularidad en las correspondencias entre magnitudes, número de cajas y número de naranjas (todas las cajas tienen el mismo número de naranjas), peso y precio (todos los kilos de naranjas valen lo mismo).

\subsection{Configuración 5 (Aplicación)}

\section{Prácticas y objetos}

Se propone aplicar el procedimiento de reducción a la unidad para resolver tres problemas de proporcionalidad directa, presentados en lenguaje natural (Figura 6). 


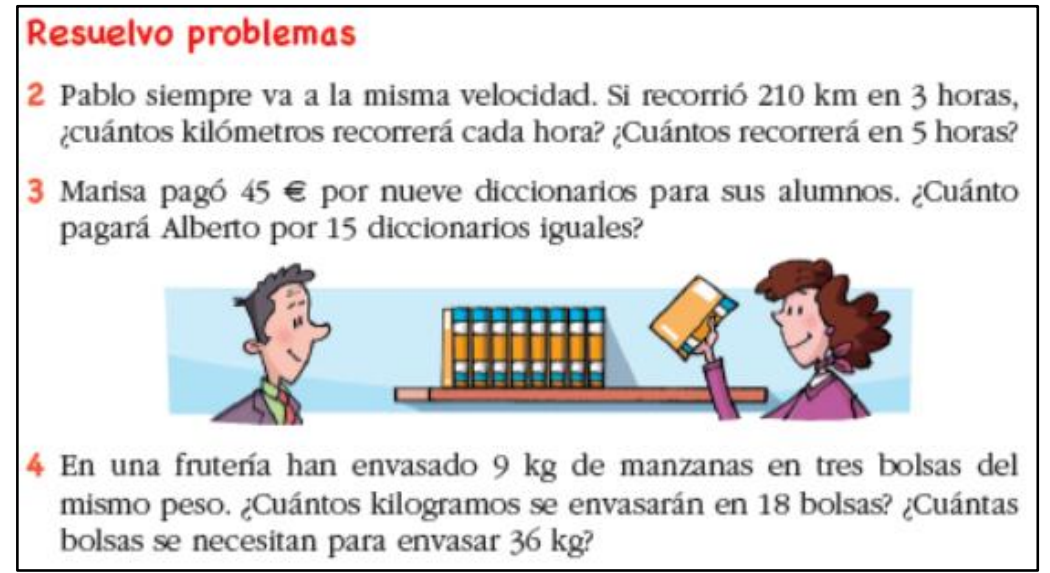

Figura 6 - Problemas de aplicación

Fuente: FERRERO et al. (2015, p. 113)

En el primer problema, se pide hallar la distancia que recorrerá cada hora. En el segundo, se pide hallar el coste de quince diccionarios, por lo que el estudiante debe hallar previamente lo que cuesta uno. A diferencia de estos dos, en el tercero se propone un problema mixto de valor perdido, en el cual la incógnita varía; es decir, en primer lugar la incógnita es el peso, y en el otro, el número de bolsas.

Se espera que el estudiante traduzca primero los enunciados a tablas de proporcionalidad (paso de lenguaje natural a lenguaje diagramático-tabular) y seguidamente calcule los valores faltantes pedidos.

Procesos

- Representación: conversión del lenguaje natural al tabular.

- Algoritmización: dominio de la técnica de reducción a la unidad.

Conflictos potenciales

Los autores han sido cuidadosos en esta ocasión, mencionando la condición de regularidad de las relaciones entre las magnitudes (misma velocidad; diccionarios iguales; mismo peso admitido por las bolsas). Posiblemente, la traducción del enunciado verbal al tabular sea conflictiva para algunos estudiantes, principalmente en lo que refiere al tercer problema dado que combina las incógnitas.

\subsection{Configuración 6 (procedimental). La regla de tres}

\section{Prácticas y objetos}

Como se muestra en la Figura 7, se comienza indicando que la regla de tres es otro procedimiento para resolver los problemas de proporcionalidad directa: consiste en calcular uno de los cuatro valores cuando se conocen los otros tres. Seguidamente, se incluye un 
ejemplo ilustrativo de la técnica basado en la escritura de una tabla de proporcionalidad, reducida a dos columnas de datos; el valor desconocido se indica con la letra $x$.

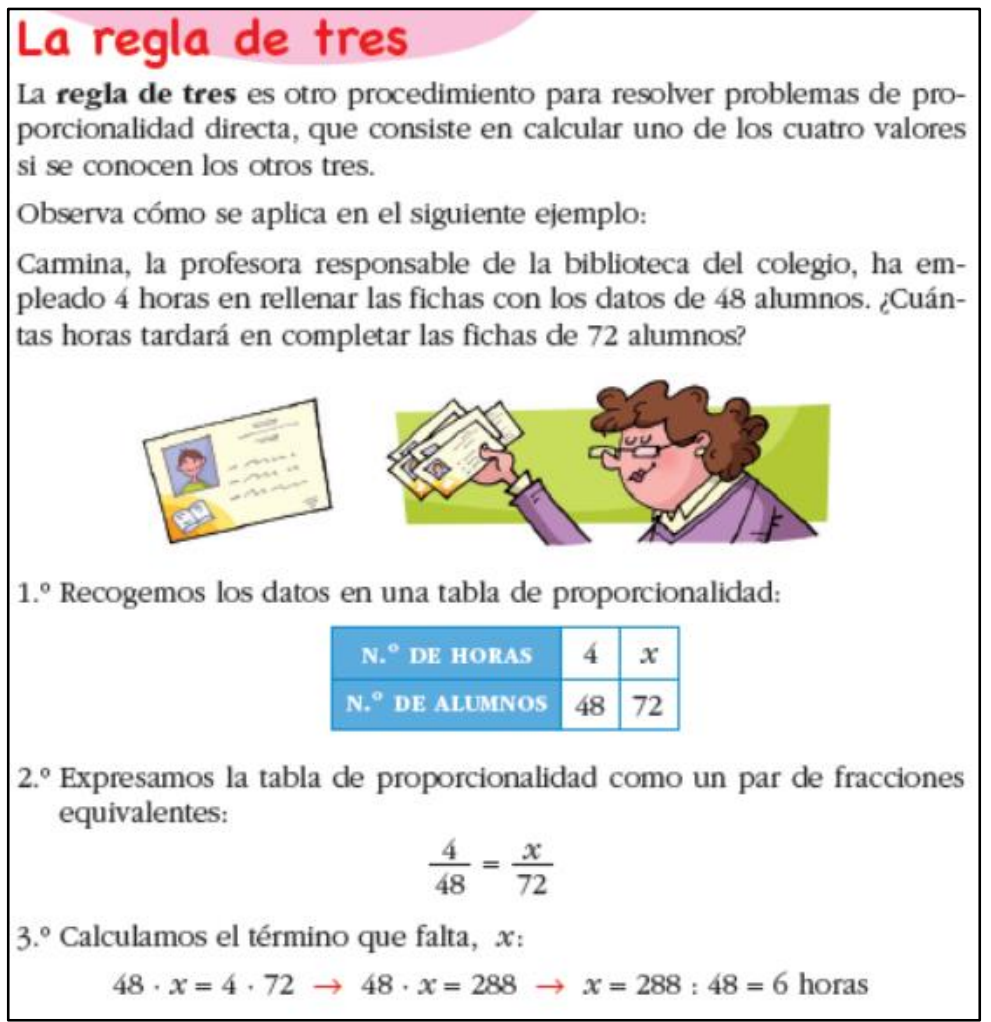

Figura 7 - La regla de tres

Fuente: FERRERO et al. (2015, p. 114)

De la conversión de las dos columnas de la tabla en una proporción (par de fracciones equivalentes), se obtiene como resultado una ecuación lineal con una incógnita.

La resolución de la ecuación se lleva a cabo, aplicando la propiedad igualdad de productos cruzados a las dos fracciones equivalentes y despejando la incógnita.

En la secuencia de prácticas operativas y discursivas descritas interviene una trama de objetos conceptuales, procedimentales y representacionales que se suponen conocidos, los cuales se articulan en el objeto procedimental emergente regla de tres. Entre ellos, están los conceptos de incógnita, ecuación, solución de una ecuación, fracciones equivalentes. Además, en la conversión de tabla a fracciones equivalentes es indispensable el concepto de razón de proporcionalidad que no se ha mencionado hasta el momento, y que constituye el argumento de este procedimiento que se muestra de manera mecánica.

\section{Procesos}

- Algoritmización: regulación de los pasos que componen el algoritmo de la regla de tres.

- Representación: conversión de los datos del problema a una tabla y conversión de tabla de proporcionalidad a equivalencia de fracciones. 
- Resolución de una ecuación.

Conflictos potenciales

Los aspectos potencialmente conflictivos del texto se refieren, básicamente, a la argumentación (explicación y justificación) que se da de la situación-problema usada como ejemplo, de la transformación entre la representación tabular y fraccionaria y la justificación de la proposición producto de medios es igual al producto de extremos.

El estudiante hipotético que leyera el texto se puede preguntar:

- ¿Por qué la relación entre el número de horas y el número de alumnos es de proporcionalidad directa? ¿Qué supuestos hay que aceptar para que el modelo de proporcionalidad directa sea aplicable?

- ¿Por qué se escriben en forma de fracción los dos pares de números que se corresponden? ¿Por qué son iguales?

- ¿Por qué se escribe la ecuación, $48 \cdot x=4 \cdot 72$ ?

\subsection{Configuración 7 (ejercitación). Encontrar el dato que falta}

\section{Prácticas y objetos}

Se proponen cuatro ejercicios (Figura 8), con datos numéricos descontextualizados, para hallar el dato representado por una letra en tablas de proporcionalidad con dos columnas.

$$
\begin{aligned}
& 1 \text { Encuentra el dato que falta en estas tablas de proporcionalidad: } \\
& \text { a) } \begin{array}{|c|c|}
\hline 5 & \mathrm{~A} \\
\hline 60 & 72 \\
\hline
\end{array} \\
& \text { b) } \begin{array}{|l|l|}
\hline 4 & 10 \\
\hline \text { B } & 15 \\
\hline
\end{array} \\
& \text { c) } \begin{array}{|c|c|}
\hline 3 & 18 \\
\hline 5 & \mathrm{C} \\
\hline
\end{array} \\
& \text { d) } \begin{array}{|c|c|}
\hline 9 & \mathrm{D} \\
\hline 12 & 25 \\
\hline
\end{array}
\end{aligned}
$$

Figura 8 - Ejercitación. Datos faltantes

Fuente: FERRERO et al. (2015, p. 113)

Se deben identificar los dos números que se multiplican y el número por el que se divide, así como interpretar las letras como incógnitas o valores faltantes en una supuesta relación de proporcionalidad.

Procesos

- Algoritmización: rutina de cálculo del valor perdido en una tabla de proporcionalidad.

- Interpretación de letras como valores faltantes.

Conflicto didáctico 
Se crea la rutina que da lugar a la regla de tres degenerada (GODINO et al., 2017, p. 6 ); en este sentido, para resolver un problema de proporcionalidad basta poner los cuatros valores en una tabla $2 \times 2$, multiplicar dos números y dividir por el tercero.

\subsection{Configuración 8 (aplicación). Serie de problemas de aplicación de la regla de tres}

\section{Prácticas y objetos}

Se proponen siete problemas contextualizados similares al mostrado en la Figura 9 para resolver aplicando la regla de tres. Se incluye, también, el siguiente enunciado, en una sección destacada como Piensa un poco: En clase hay cuatro chicas más que chicos. ¿Cuántos chicos y chicas hay si en total somos 24? Dado que la relación entre el número de chicos y chicas es aditiva este enunciado permite reflexionar sobre el carácter multiplicativo que caracterizan las relaciones de proporcionalidad. Se espera que el alumno plantee la ecuación $x+(4+x)=24 ; y$ despeje el valor de $x=10$.

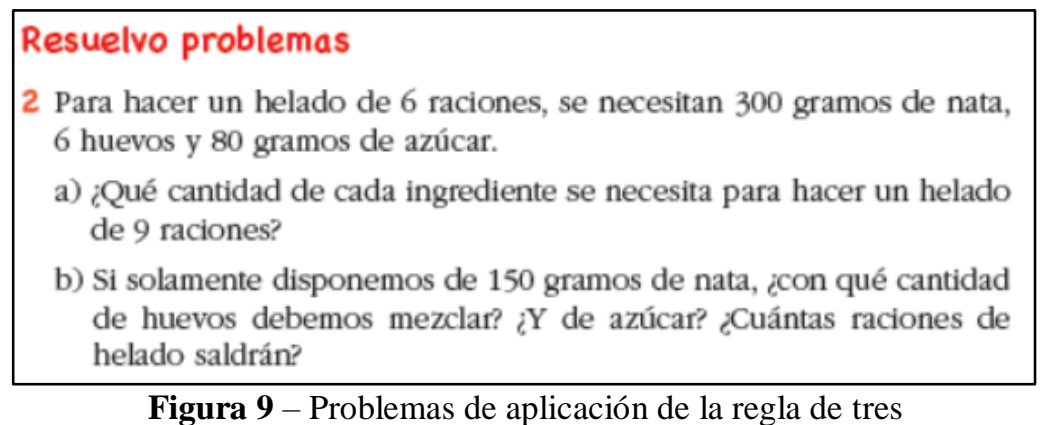
Fuente: FERRERO et al. (2015, p. 113)

\section{Procesos}

- Interpretación de los enunciados, representación (conversión del registro de la lengua natural al registro tabular; tratamientos en el registro numérico para hallar valores faltantes en las tablas de proporcionalidad).

- Algoritmización: aplicación de la técnica de la regla de tres.

- Resolución de problemas.

Conflictos potenciales

- Dado que no se ha pedido, a lo largo de la lección, reflexionar sobre el carácter lineal o no lineal de las situaciones-problemas es previsible que se esté generando el conflicto de ilusión de linealidad (DE BOCK; VERSCHAFFEL; JANSEENS, 1998; VAN DOOREN et al., 2008), que responde a la tendencia a utilizar modelos lineales en situaciones no pertinentes para su aplicación. 
- Todos los ejemplos anteriores y ejercicios tratados involucran dos magnitudes, en este caso se están considerando más gramos de nata, cantidad de huevos, gramos de azúcar. Al representar esto en una tabla es diferente; un alumno podría preguntarse ¿cómo dispongo las filas y las columnas?, ¿debo hacer varias tablas o una es suficiente?, ¿si solo hago una, existen muchas incógnitas, cómo procedo?

\section{Faceta instruccional}

La faceta instruccional incluye, en el marco del EOS, las interacciones entre docente y discentes o entre discentes y el uso de recursos didácticos, entre los cuales se incluye el tiempo asignado al desarrollo del tema.

En el caso de una lección de un libro de texto, el autor (en su papel de docente) presenta la información, incluyendo las tareas (ejercicios y problemas) que debe realizar el lector (estudiante). Bajo este esquema, el modelo instruccional es esencialmente de tipo objetivista/transmisivo (GODINO et al., 2016; GODINO et al., 2019b).

La implementación de un modelo constructivista/indagativo, centrado en la construcción del conocimiento por parte del estudiante, no parece posible si el docente diseña y planifica el proceso de instrucción en base únicamente a un libro de texto, cuando se trata de lecciones escritas con un formato de libro tradicional. La primera fase de un modelo constructivista, como puede ser el propuesto por la Teoría de Situaciones Didácticas en Matemáticas (TSDM) (BROUSSEAU, 1997), consistente en la presentación de las consignas de una situación-problema, sería posible de implementar. Pero las siguientes fases de trabajo autónomo del estudiante (momentos de exploración, formulación, comunicación y validación) no pueden quedar plasmadas en un libro de texto.

Esencialmente, el libro centra la atención en lo que podríamos llamar la fase o momentos de institucionalización, donde se presentan y fijan los conocimientos institucionales pretendidos. Parece imposible articular, de manera interactiva, la trayectoria epistémica (secuencia de los conocimientos institucionales pretendidos) con la trayectoria cognitiva (secuencia de los aprendizajes de los estudiantes).

El modelo instruccional característico de los libros de texto parece que está restringido a lo que podríamos describir como: primero teoría, después, ejemplos, ejercicios y aplicaciones. Este es el modelo que hemos podido apreciar en la secuencia de configuraciones (trayectoria didáctica) de la lección de proporcionalidad del libro que analizamos. Incluso la teoría (el contenido) queda restringido a algunos aspectos conceptuales y una fuerte dosis de 
procedimientos rutinarios, siendo prácticamente ausentes las justificaciones de los procedimientos y proposiciones que intervienen.

En Godino et al. (2016) y Godino et al. (2019b) se analiza la tensión dialéctica existente entre los modelos instruccionales de tipo objetivista y los constructivista, argumentando a favor de un modelo de tipo mixto en el que el diálogo, la cooperación, el trabajar conjuntamente en la solución de tareas problemáticas puede incrementar la idoneidad didáctica del proceso instruccional, cuando se asumen presupuestos de tipo sociocultural (LERMAN, 2001; RADFORD, 2011) sobre el aprendizaje matemático.

Bajo esta perspectiva, el libro de texto podría ser usado como recurso que facilite la implementación de ese modelo instruccional de tipo mixto. La presentación de ejemplos de problemas resueltos en los cuales se contextualicen los conocimientos pretendidos puede ser más efectiva que la exploración autónoma por los propios estudiantes.

Como afirman Sweller, Kirschener y Clark (2007, p. 118),

[...] debemos aprender soluciones específicas del dominio para problemas específicos y la mejor manera de adquirir estrategias de resolución de problemas específicas de un dominio es dar el problema con su solución, no dejando ningún papel al aprendizaje basado en la indagación.

Estos autores afirman que la investigación empírica del último medio siglo, sobre este problema, proporciona una abrumadora y clara evidencia de que una mínima guía durante la instrucción es significativamente menos efectiva y eficiente que una guía específicamente diseñada para apoyar el procesamiento cognitivo necesario para el aprendizaje. Resultados similares se reflejan en el meta-análisis de Alfieri et al. (2011).

\section{Síntesis de hechos didácticos significativos}

Un aspecto importante que se debe considerar es el orden que sugiere el libro de texto con respecto a la secuencia de los conocimientos y competencias matemáticas pretendidas. Tal como se muestra en la sección 5, en las unidades de análisis se identifican con ocho tipos de configuraciones:

- Configuración 1 (conceptualización)

- Configuración 2 (ejercitación)

- Configuración 3 (procedimental)

- Configuración 4 (ejercitación)

- Configuración 5 (aplicación)

- $\quad$ Configuración 6 (procedimental) 


\section{- $\quad$ Configuración 7 (ejercitación) \\ - Configuración 8 (aplicación)}

El hecho de que la primera configuración sea de conceptualización choca con el consenso que parece existir en la comunidad de educadores e investigadores acerca de que lo ideal sería la enseñanza a través de la resolución de problemas. Se espera que la resolución de problemas sea, no solo el objetivo fundamental del aprendizaje de las matemáticas, sino también que constituya el eje vertebrador sobre el que articular la totalidad de los contenidos establecidos por el currículo. Gaulin (2001) describe las tres opciones de enseñanza que puede considerar un docente con relación al papel de la resolución de problemas:

- Enseñanza para resolver problemas;

- Enseñanza a través de la resolución de problemas;

- Enseñanza sobre resolución de problemas.

Otros autores, como Castro (2008), se hacen eco de estas opciones de enseñanza, y de las ideas, tendencias e influencias en la resolución de problemas en España. English y Gainsburg (2016) reflexionan acerca de la importancia del contenido matemático per se y de las expectativas de la sociedad. Como punto de encuentro, recomiendan la utilización de problemas ricos, que precisen de la utilización de contenido curricular.

En la configuración 3, se comienza describiendo, de manera general, el procedimiento, sin describir previamente los problemas que se quieren resolver dejando a la vista una enseñanza procedimental centrada en resolver ejercicios y no a través de la resolución de problemas.

Se resalta el hecho de que no se traten situaciones que hagan reflexionar sobre si la relación en una situación-problema es multiplicativa o aditiva. De acuerdo con Dole y Shield (2008, p. 20) "ser capaz de distinguir entre situaciones aditivas y multiplicativas es la base para poder determinar situaciones proporcionales y no proporcionales".

Las situaciones-problemas que se trabajan en el texto suelen referir al enfoque aritmético, y los problemas suelen ser de comparación y valor faltante. Sin embargo, se echa en falta la existencia de estrategias progresivas para avanzar de lo aditivo a lo multiplicativo. También, debe cuidarse la redacción de las condiciones en los problemas, señalando, por ejemplo, si las cantidades de litros por botellas son las mismas.

No se trabaja la proporcionalidad con los otros enfoques antes mencionados, que son relevantes para abordar el tema de una manera global. Este hecho, unido con las expectativas de aprendizaje según el MECD (2014) evidencia carencias en la propuesta. Sin embargo, se 
debe destacar que se proponga el uso de tablas y ecuaciones, así como el uso de números naturales pequeños.

Tampoco se generaliza la linealidad $y=k x$; se dan por conocidos varios conceptos relevantes y no se menciona el concepto de constante de proporcionalidad el cual es imprescindible para entender el paso que se hace de la representación tabular a fracciones equivalentes relatada en la configuración 6. Además, como señalamos anteriormente, según Lamon (2007) el reconocimiento de la razón constante es la clave para comprender el razonamiento proporcional; tampoco el concepto de razón se menciona o trabaja.

La introducción de la regla de tres desprovista de argumentos que la sustenten puede desencadenar conflictos y razonamientos inadecuados, incentivando a aplicar el procedimiento como una rutina.

Ante ese conflicto, se agrega que la solución de las situaciones-problemas también puede darse mediante una vinculación con el tema de fracciones equivalentes, haciendo uso de simplificación o amplificación de fracciones según sea el caso. De este modo, se evidencia que la resolución de un problema puede hacerse de diversas formas, poniendo en juego, según las estrategias usadas, diferentes niveles de algebrización.

Por último, en el análisis se destaca un fuerte vínculo entre los procesos de representación y los métodos de resolución propuestos por los problemas; por ejemplo, considerando la instrucción en las configuraciones precedentes, en la configuración 5 se refleja que la representación tabular condiciona el método de resolución del problema planteado. Resultado ya señalado por Martínez-Juste et al. (2017) cuando analizan la proporcionalidad en libros de texto de nivel secundario.

\section{Comentarios finales e implicaciones}

Hemos presentado un método para analizar lecciones de libros de texto aplicando las herramientas teóricas del EOS, ejemplificado en el caso de una lección sobre proporcionalidad de un libro de sexto curso de primaria. Se han tenido en cuenta las facetas y componentes que la Teoría de la Idoneidad Didáctica propone para el análisis de un proceso de instrucción matemática.

Se parte del supuesto de que una lección de un libro de texto constituye la planificación de un proceso de instrucción en el que un estudiante hipotético usa el texto para abordar el estudio del contenido correspondiente. 
El texto propone una trayectoria epistémica, esto es, la selección y secuencia de un sistema de prácticas operativas, discursivas y normativas, implicadas en la realización de una muestra de tareas (ejemplos, ejercicios, problemas de aplicación). La trayectoria cognitiva del estudiante hipotético (lector), que refleja la progresión del aprendizaje, solo es posible describirla en el componente parcial de los conocimientos previos requeridos. La confrontación de la trayectoria epistémica y cognitiva, cuando se contrasta con los conocimientos didáctico-matemáticos disponibles sobre el tema, permite identificar potenciales conflictos, información que puede ser de utilidad en la planificación de las trayectorias didácticas que, efectivamente, puede implementar un docente que use el libro de texto.

Cuando un docente usa la lección como recurso se abre un panorama más rico y complejo para el análisis didáctico, ya que el docente puede modificar la trayectoria epistémica prevista en la lección y articularla con las trayectorias cognitivas de sus estudiantes. También, puede incorporar el uso de recursos manipulativos y tecnológicos complementarios, así como gestionar adecuadamente el tiempo y la evaluación progresiva de los aprendizajes.

El modelo instruccional básicamente objetivista-transmisivo, inherente a la presentación del contenido que hace el libro de texto tradicional, puede evolucionar con la intervención del profesor hacia un modelo mixto en el que la solución de las situacionesproblemas introductorias que propone el texto puede hacerse de manera dialógica y cooperativa, incrementando la idoneidad instruccional de las trayectorias didácticas efectivamente implementadas. Nuestro método de análisis puede ayudar a abordar el tema del uso que deberían hacer los profesores del libro de texto (FAN; ZHU; MIAO, 2013), y la consiguiente línea de investigación sobre formación de profesores.

La realización del tipo de análisis didáctico detallado de las configuraciones didácticas que hemos presentado en este trabajo debería ser una competencia del profesor de matemáticas, formando parte de la competencia más general de planificación de lecciones. El diseño de talleres formativos sobre este tema es una cuestión abierta a nuevas investigaciones en el campo de la formación de profesores. También, es una cuestión abierta el diseño y evaluación de lecciones basadas en el uso de hipertextos y otros recursos tecnológicos que permiten implementar trayectorias epistémicas, cognitivas e instruccionales más interactivas que las permitidas por los libros de texto tradicionales. 


\section{Reconocimiento}

Trabajo realizado en el marco de los proyectos de investigación PID2019-105601GBI00 y PGC2018-098603-B-I00 (Ministerio de Ciencia, Innovación y Universidades, España), Grupo FQM126 (Junta de Andalucía) y Grupo S36_17D Investigación en Educación Matemática (Gobierno de Aragón y Fondo Social Europeo), España.

\section{Referencias}

ALFIERI, L.; BROOKS, P.J.; ALDRICH, N.J.; TENENBAUM, H.R. Does discovery-based instruction enhance learning? Journal of Educational Psychology, Washington, v. 103, n. 1, p. 1-18, 2011.

AMERICAN ASSOCIATION FOR THE ADVANCEMENT OF SCIENCE (AAAS). Middle grades mathematics textbooks: A benchmarks-based evaluation, 2000. Disponible en:

http://www.project2061.org/publications/textbook/mgmth/report/default.htm . Acceso en: 01 mar. 2019.

AROZA, C. J.; GODINO, J. D.; BELTRÁN-PELLICER, P. Iniciación a la innovación e investigación educativa mediante el análisis de la idoneidad didáctica de una experiencia de enseñanza sobre proporcionalidad. AIRES, Granada, v. 6, n. 1, 1-29, 2016.

BREDA, A.; FONT, V.; PINO-FAN, L. R. Criterios valorativos y normativos en la Didáctica de las Matemáticas: el caso del constructo idoneidad didáctica. Bolema, Rio Claro, v. 32, n. 60, p. 255-278, 2018.

BREDA, A.; PINO-FAN, L.; FONT, V. Meta Didactic-Mathematical Knowledge of Teachers: Criteria for the reflection and assessment on teaching practice. EURASIA - Journal of Mathematics Science and Technology Education, London, v. 13, n. 6, p. 1893-1918, 2017.

BROUSSEAU, G. The theory of didactical situations in mathematics. Dordrecht: Kluwer, 1997.

CASTRO, E. Resolución de problemas: ideas, tendencias e influencias en España. In: LUENGO, R.; GÓMEZ, B.; CAMACHO, M.; BLANCO, L. (Ed.). Investigación en educación matemática XII. Badajoz: SEIEM, 2008. p. 113-140.

DE BOCK, D.; VERSCHAFFEL, L.; JANSSENS, D. The predominance of the linear model in secondary school students' solutions of word problems involving length and area of similar plane figures. Educational Studies in Mathematics, Utrecht, v. 35, p. 65-85, 1998.

DOLE, S.; SHIELD, M. The capacity of two Australian eight-grade textbooks for promoting proportional reasoning. Research in Mathematics Education, Barcelona, v. 10, n. 1, p. 19-35, 2008.

ENGLISH, L. D.; GAINSBURG, J. Problem Solving in a 21st-Century Mathematics Curriculum. In: ENGLISH, L. D.; KIRSHNER, D. (Ed.). Handbook of International Research in Mathematics Education. New York: Routledge, 2016. p. 313-335.

FAN, L.; ZHU, Y.; MIAO, Z. Textbook research in mathematics education: development status and directions. ZDM Mathematics Education, Berlín, v. 45, n. 5, p. 633-646, sep. 2013. 
FERNÁNDEZ, A. Precursores del racionamiento proporcional: un estudio con alumnos de primaria. 2001. Tesis (Doctorado en Ciencias de la Educación) Universidad de Valencia, Valencia, 2001. Disponible en: https://dialnet.unirioja.es/servlet/tesis?codigo=81910. Acceso en: 01 mar. 2019.

FERNÁNDEZ, C.; LLINARES, S. Características del desarrollo del razonamiento proporcional en la Educación Primaria y Secundaria. Enseñanza de las Ciencias, Barcelona, v. 30, n. 1, p. 129-142, 2012.

FERRERO, L.; MARTÍN, P.; ALONSO, G.; BERNAL, E. I. Matemáticas 6. Madrid: Anaya. 2015.

FIOL, M. L.; FORTUNY, J. M. Proporcionalidad directa: La forma y el número. Madrid: Síntesis, 1990.

FONT, V.; GODINO, J. D. La noción de configuración epistémica como herramienta de análisis de textos matemáticos: su uso en la formación de profesores. Educaçao Matemática Pesquisa, São Paulo, v. 8, n. 1, p. 67-98, 2006.

GASCÓN, J.; BOSCH, M.; RUIZ-MUNZÓN, N. El problema del álgebra elemental en la teoría antropológica de lo didáctico. In: MUÑOZ-ESCOLANO, J. M.; ARNAL-BAILERA, A.; BELTRÁN-PELLICER, P.; CALLEJO, M. L.; CARRILLO, J. (Ed.). Investigación en Educación Matemática XXI. Zaragoza: SEIEM, 2017. p. 25-47.

GAULIN, C. Tendencias actuales de la resolución de problemas. Sigma, Vitoria, v. 19, p. 51-63, 2001.

GEA, M. M. La correlación y regresión en bachillerato: análisis de libros de texto y del conocimiento de los futuros profesores. Tesis (Doctorado en Ciencias de la Educación) - Facultad de Educación, Universidad de Granada, Granada, 2014.

GODINO, J. D. Indicadores de la idoneidad didáctica de procesos de enseñanza y aprendizaje de las matemáticas. Cuadernos de Investigación y Formación en Educación Matemática, Costa Rica, v. 11, p. 111-132, 2013.

GODINO, J. D. Construyendo un sistema modular e inclusivo de herramientas teóricas para la educación matemática. En J. M. CONTRERAS, P. ARTEAGA, G. R. CAÑADAS, M.M. GEA, B. GIACOMONE Y M. M. LÓPEZ-MARTÍN (Ed), Actas del Segundo Congreso International Virtual sobre el Enfoque Ontosemiótico del Conocimiento y la Instrucción Matemáticos. Granada, 2017. Disponible en: http://enfoqueontosemiotico.ugr.es/civeos.html.

GODINO, J. D.; AKÉ, L.; GONZATO, M.; WILHELMI, M. R. Niveles de algebrización de la actividad matemática escolar. Implicaciones para la formación de maestros. Enseñanza de las Ciencias, Barcelona, n. 1, p. 199-219, 2014a.

GODINO, J. D.; BATANERO, C; CAÑADAS, G.; CONTRERAS, J. M. Linking inquiry and transmission in teaching and learning mathematics and experimental sciences. Acta Scientiae, Capes, v. 18 , n. 4, p. 29-47, 2016.

GODINO, J. D.; BATANERO, C.; FONT, V. The onto-semiotic approach to research in mathematics education. ZDM. The International Journal on Mathematics Education, Heidelberg, v. 39, n. 1-2, p. 127-135, 2007.

GODINO, J. D.; BATANERO, C.; FONT, V. The onto-semiotic approach: implications for the prescriptive character of didactics. For the Learning of Mathematics, New Westminster, v. 39, n. 1, p. 37- 42, 2019a. 
GODINO, J. D.; BELTRÁN-PELLICER, P.; BURGOS, M.; GIACOMONE, B. Significados pragmáticos y configuraciones ontosemióticas en el estudio de la proporcionalidad. In: CONTRERAS, J. M.; ARTEAGA, P.; CAÑADAS, G. R.; GEA, M. M.; GIACOMONE, B.; LÓPEZMARTÍN, M. M. (Ed.). Actas del Segundo Congreso International Virtual sobre el Enfoque Ontosemiótico del Conocimiento y la Instrucción Matemáticos. Granada: CIVEOS, 2017. p. 1-13.

GODINO, J. D.; BURGOS, M. Perspectiva ontosemiótica del razonamiento algebraico escolar. In: MUÑOZ-ESCOLANO, J. M.; ARNAL-BAILERA, A.; BELTRÁN-PELLICER, P.; CALLEJO, M. L.; CARRILLO, J. (Ed.). Investigación en Educación Matemática XXI. Zaragoza: SEIEM, 2017. p. 49-66.

GODINO, J. D.; CONTRERAS, A.; FONT, V. Análisis de procesos de instrucción basado en el enfoque ontológico-semiótico de la cognición matemática. Recherches en Didactiques des Mathématiques, Grenoble, v. 26, n. 1, p. 39-88, 2006.

GODINO, J. D.; FONT, V.; WILHELMI, M. R. Análisis ontosemiótico de una lección sobre la suma y la resta. RELIME -Revista Latinoamericana de Investigación en Matemática Educativa, Ciudad de Mexico, v. 9 (Especial), p. 131-155, 2006.

GODINO, J. D.; FONT, V.; WILHELMI, M. R.; CASTRO, C. de. Aproximación a la dimensión normativa en Didáctica de la Matemática desde un enfoque ontosemiótico. Enseñanza de las Ciencias, Barcelona, v. 27, n. 1, p. 59-76, 2009.

GODINO J. D.; RIVAS H.; ARTEAGA P.; LASA A.; WILHELMI M. R. Ingeniería didáctica basada en el enfoque ontológico-semiótico del conocimiento y la instrucción matemáticos. Recherches en Didactique des Mathématiques, Grenoble, v. 34, n. 2/3, p. 167-200, 2014b.

GODINO, J. D.; RIVAS, H.; BURGOS, M.; WILHELMI, M. D. Analysis of didactical trajectories in teaching and learning mathematics: overcoming extreme objectivist and constructivist positions. International Electronic Journal of Mathematics Education, Ankara, v. 14, n. 1, p. 147-161, $2019 b$.

INHELDER, B.; PIAGET, J. The growth of logical thinking from childhood to adolescence. New York: Basic Books, 1958.

KARPLUS, R.; PULOS, S.; STAGE, E. Early adolescents' proportional reasoning on "rate" problems. Educational Studies in Mathematics, Utrecht, v. 14, p. 219-233, 1983.

LAMON, S. Rational number and proportional reasoning: toward a theoretical framework for research. En Lester. F. K. (Ed.), Second handbook of research on mathematics teaching and learning. Charlotte, NC: Information Age Publishing, v. 1, p. 629-667, 2007.

LERMAN, S. Cultural, discursive psychology: a sociocultural approach to studying the teaching and learning of mathematics. Educational Studies in Mathematics, Utrecht, v. 47, p. 87-113, 2001.

MARTÍNEZ JUSTE, S.; MUÑOZ ESCOLANO, J. M.; OLLER MARCÉN, A. M.; ORTEGA DEL RINCÓN, T. Análisis de problemas de proporcionalidad compuesta en libros de texto de $2^{\circ}$ de ESO. Revista Latinoamericana de Investigación en Matemática Educativa, Ciudad de Mexico, v. 20, n. 1, p. 95-122, 2017.

MINISTERIO DE EDUCACIÓN, CULTURA Y DEPORTE. Real Decreto 126/2014, de 28 de febrero, por el que se establece el currículo básico de la Educación Primaria. Madrid: MECD, 2014.

NATIONAL COUNCIL OF TEACHERS OF MATHEMATICS. Principles and standards for school mathematics. Reston: The National Council of Teachers of Mathematics, 2000. 
ORTIZ DE HARO, J. J. Significado de los conceptos probabilísticos en los libros de texto de Bachillerato. Tesis doctoral. Universidad de Granada, 1999. Disponible en:

https://www.ugr.es/ batanero/documentos/tesisjj.PDF. Acceso en: 01 mar. 2019.

RADFORD, L. La evolución de paradigmas y perspectivas en la investigación. El caso de la didáctica de las matemáticas. In VALLÈS, J.; ÁLVAREZ, D.; RICKENMANN, R. (Ed.). L'ctivitat docent intervenció, innovació, investigació. Girona: Documenta Universitaria, 2011. p. 33-49.

STREEFLAND, L. Search for roots of ratio: some thoughts on the long-term learning process (towards... a theory) part II: the outline of the long-term learning process. Educational Studies in Mathematics, Utrecht, v. 16, n. 1, p. 75-94, 1985.

SWELLER J.; KIRSCHNER P. A.; CLARK R. E. Why minimally guided teaching techniques do not work: A reply to commentaries. Educational Psychologist, Washington, v. 42, n. 2, p. 115-121, 2007.

TOURNIAIRE, F. Y PULOS, S. Proportional reasoning: A review of the literature. Educational Studies in Mathematics, Utrecht, v. 16, p. 181-204, 1985.

VAN DOOREN, W.; DE BOCK, D.; JANSSENS, D.; VERSCHAFFEL, L.The linear imperative: An inventory and conceptual analysis of students' over-use of linearity. Journal for Research in

Mathematics Education, Reston VA, v. 39, n. 3, p. 311-342, 2008.

WILHELMI, M. R. Proporcionalidad en Educación Primaria y Secundaria. In J. M. CONTRERAS; P. ARTEAGA; G. R. CAÑADAS; M. M. GEA; B. GIACOMONE; M. M LÓPEZ MARTÍN (Ed.). Actas del Segundo Congreso Internacional Virtual sobre el Enfoque Ontosemiótico del Conocimiento y la Instrucción Matemáticos. 2017a. Disponible en: enfoqueontosemiotico.ugr.es/civeos.html. Acceso en: 01 mar. 2019.

WILHELMI, M. R. Didáctica del Álgebra. En MUÑOZ-ESCOLANO, J. M.; ARNAL-BAILERA, A.; BELTRÁN-PELLICER, P.; CALLEJO, M. L.; CARRILLO, J. (Ed.). Investigación en Educación Matemática XXI. Zaragoza: SEIEM, 2017b. p. 17-23. 